\title{
Joint Transmitter-Receiver Frequency-Domain Equalization in Generalized Multicarrier Code-Division Multiplexing Systems
}

\author{
Tingting Liu, Student Member, IEEE, Chenyang Yang, Senior Member, IEEE, and \\ Lie-Liang Yang, Senior Member, IEEE
}

\begin{abstract}
Joint transmitter-receiver optimization in generalized multicarrier code-division multiplexing (GMC-CDM) systems is investigated in this paper. The optimization consists of a one-tap post-frequency-domain equalizer (post-FDE) and a onetap pre-FDE. While the one-tap post-FDE is optimized under the criterion of minimum mean square error (MMSE), the one-tap pre-FDE is achieved through three stages of optimization, which are operated at different levels and motivated to achieve, possibly, different objectives, including maximum throughput and maximum reliability. Specifically, in our three-stage pre-FDE, the first-stage pre-FDE is operated at the symbol level, concerning only the symbols within a group. The second-stage pre-FDE is carried out at the group level for harmonization among the groups. Finally, the third-stage pre-FDE handles group partition. In this paper, the error and throughput performance of the GMC-CDM systems is investigated when assuming communications over frequency-selective Rayleigh fading channels. It can be shown that the reliability or throughput of the GMC-CDM systems can be significantly improved by employment of the proposed pre- and post-FDE schemes. Furthermore, the pre- and post-FDE algorithms obtained can be implemented with high flexibility, which facilitates a GMC-CDM system to achieve a good tradeoff between its throughput and reliability.
\end{abstract}

Index Terms-Frequency-domain equalization (FDE), joint transmitter-receiver optimization, multicarrier code-division multiple access (MC-CDMA), power allocation, preprocessing.

\section{INTRODUCTION}

$\mathbf{M}$ ULTICARRIER techniques are promising for broadband communications, since they are capable of providing high spectral efficiency and high flexibility with low-complexity transceivers, which only require one-tap frequency-domain equalization (FDE) [1]. Multicarrier codedivision multiple access (MC-CDMA) is capable of achieving frequency diversity by spreading signals to be transmitted over

Manuscript received November 13, 2009; revised April 22, 2010; accepted June 27, 2010. Date of publication July 12, 2010; date of current version October 20, 2010. This work was supported in part by the International S\&T Cooperation Program of China under Grant 2008DFA12100. This paper was presented in part at the 2009 IEEE Vehicular Technology Conference, Anchorage, AK, September 20-23, 2009. The review of this paper was coordinated by $\mathrm{H}$. H. Nguyen.

T. Liu and C. Yang are with the School of Electronics and Information Engineering, Beihang University, Beijing 100191, China (e-mail: ttliu@ee. buaa.edu.cn; cyyang@buaa.edu.cn).

L.-L. Yang is with the School of Electronics and Computer Science, University of Southampton, Southampton SO17 1BJ, U.K. (e-mail: 1ly@ecs. soton.ac.uk).

Digital Object Identifier 10.1109/TVT.2010.2057266 multiple subcarriers experiencing frequency-selective fading. However, when communicating over frequency-selective fading channels, MC-CDMA may suffer from multiuser interference (MUI) and intersymbol interference if multiple users are supported, and each user transmits multiple symbols using code-division multiplexing (CDM) [2]-[6]. In MC-CDMA systems, if channel-state information (CSI) is known to the transmitter, which is referred to as the CSIT mode, the system performance may be improved with the aid of transmitter preprocessing carried out at the transmitter side [2]. However, when there is no cooperation among the transmitters, the design of transmitter-receiver processing algorithms is usually highly involved, and often, no closed-form solutions are available [3]-[5].

In [7]-[9], a generalized MC-CDMA (GMC-CDMA) scheme has been proposed, which is also referred to as the orthogonal frequency CDMA. In the GMC-CDMA scheme, multiple users are separated in the frequency domain, whereas the different data streams of a user are transmitted in the principles of both frequency-division multiplexing (FDM) and CDM. It has been shown that the GMC-CDMA scheme can inherit the advantages of both orthogonal frequency-division multipleaccess (OFDMA) and MC-CDMA schemes [7], [9]-[11]. It is MUI free as the OFDMA scheme while attaining frequency diversity as the MC-CDMA. In GMC-CDMA systems, the multiple-access channels are equivalent to a number of independent parallel single-user channels [9]. Hence, the design of transceivers in GMC-CDMA systems can be significantly simplified, and the transmitter-receiver optimization is equivalent to that in the GMC-CDM systems supporting single users.

Joint transmitter-receiver optimization has been extensively studied in recent years. However, the considered optimization has been mainly motivated for achieving the single optimization objective of either maximum reliability (lowest error probability, highest signal-to-interference-and-noise ratio (SINR), etc.) or maximum throughput, as shown, e.g., in [12]-[14]. In wireless communications experiencing frequency-selective fading, as shown in [14], the aforementioned two optimization objectives usually result in totally inconsistent solutions. When the optimization motivates to maximize the throughput, FDM is the optimum transmission scheme, which achieves the highest possible throughput with the aid of water filling [15] carried out at the transmitter. By contrast, when the optimization aims 
to maximize the reliability, $\mathrm{CDM}$ is then highly desirable. This is because in CDM, each signal is transmitted on all the subcarriers, and hence, the highest frequency diversity gain can be attained. In practice, however, it is usually more attractive for a communication scheme to attain a good tradeoff between throughput and reliability, which can be adjusted correspondingly according to the variation of communications environments. In [16], a hybrid FDM-CDM scheme has been proposed, which switches between the FDM mode and the CDM mode when necessary. However, switching between two fixed modes may still not provide sufficient flexibility in comparison with the GMC-CDM systems. In GMC-CDM systems, optimizations can be implemented at several levels and are possibly for different objectives, as shown in our forthcoming discourse.

In this paper, both the one-tap pre-FDE operated at the transmitter and the one-tap post-FDE carried out at the receiver are investigated. While the one-tap post-FDE is simply optimized based on the principles of minimum mean square error (MMSE) [17], our focuses are mainly on the one-tap pre-FDE optimization. Our pre-FDE optimization procedure is divided into three stages. The first-stage pre-FDE is operated at the symbol level concerning only the symbols within a group. The second-stage pre-FDE is carried out at the group level to harmonize among the groups. Finally, the third-stage pre-FDE handles group partition (GP). In our pre-FDE optimization, the first-stage pre-FDE aims to achieve the highest possible SINR for the symbols belonging to a group. By contrast, at the second-stage pre-FDE and the third-stage GP, the optimization is either motivated to achieve a high throughput counted by the sum data rate (SDR) or targeted to attain a high SINR for improving the communications reliability. From the aforementioned three stages of optimization, we find that the resultant pre-FDE can be implemented by a simple subcarrier grouping algorithm associated with a power-allocation algorithm. The pre-FDE algorithm has a similar complexity as the classical water-filling algorithm [15]. Therefore, the one-tap pre-FDE and the one-tap post-FDE derived in this paper are of relatively low complexity. However, our studies and performance results show that they are capable of significantly enhancing the throughput or reliability of the GMC-CDM systems.

Note that the post-FDE and pre-FDE algorithms obtained can be readily extended for application in the GMC-CDMA systems that support multiple users. In GMC-CDMA systems, users are separated in the frequency domain, and there is no MUI among users. Hence, all the optimization approaches proposed in this paper may be directly applied to this type of GMC-CDMA systems. Furthermore, in GMC-CDMA systems, a further stage of equalization operated at the user level may be invoked, which can be optimized in the same way as the GP algorithm proposed in this paper, if cooperation among users is possible.

The reminder of this paper is organized as follows: Section II considers the system model and formulates the problem. Then, in Section III, post-FDE is addressed. Section IV focuses on transmitter optimization, including various pre-FDE schemes. Performance results are provided in Section V, and finally, in Section VI, we summarize the conclusions.

\section{Signal Models}

The GMC-CDM system considered in this paper employs $M$ orthogonal subcarriers and transmits $K(K \leq M)$ data streams in parallel. Hence, the spreading factor (SF) of the GMCCDM system is $N=M / K$. During a given symbol period, the signals to be transmitted in the frequency domain can be represented in matrix form as

$$
s=W_{\mathrm{pre}} d=P G_{\mathrm{pre}} U \boldsymbol{d}
$$

where the vectors and matrices are summarized as follows:

$s \quad M$-length vector denoting the signals to be transmitted on the $M$ subcarriers;

$\boldsymbol{d} \quad \boldsymbol{d}=\left[d_{0}, d_{1}, \ldots, d_{K-1}\right]^{T}$, which contains the $K$ data symbols to be transmitted. It is assumed that the entries of $\boldsymbol{d}$ are independent and identically distributed random variables satisfying $\left|d_{k}\right|^{2}=1$ for $k=0,1, \ldots, K-1$;

$\boldsymbol{W}_{\text {pre }}(M \times K)$ preprocessing matrix, which includes the transmitter processing operations of GMC-CDM, one-tap pre-FDE, and frequency-domain spreading. Therefore, it can be expressed as $\boldsymbol{W}_{\text {pre }}=\boldsymbol{P} \boldsymbol{G}_{\mathrm{pre}} \boldsymbol{U}$; $\boldsymbol{P} \quad(M \times K)$ spreading matrix, which spreads the $K$ data symbols after pre-FDE onto the $M$ subcarriers; $\boldsymbol{G}_{\text {pre }} \quad \boldsymbol{G}_{\text {pre }}=\operatorname{diag}\left\{G_{\text {pre }}^{(0)}, \ldots, G_{\text {pre }}^{(K-1)}\right\}$, which is a $(K \times$ $K)$ diagonal matrix for carrying out one-tap preFDE. Let $\Omega_{j}=\left|G_{\text {pre }}^{(j)}\right|^{2}$. Then, we have $\sum_{j=0}^{K-1} \Omega_{j} \leq$ $K$, due to the constraint on the transmission power;

$\boldsymbol{U} \quad(K \times K)$ unitary matrix implementing both CDM and FDM.

In the GMC-CDM system considered, the $K$ data symbols are first divided into $D$ groups, which are supported in the FDM principles. Then, each of the data symbols in a group is assigned an orthogonal code for implementing CDM. Hence, the multiplexing matrix $\boldsymbol{U}$ in (1) can be structured as $\boldsymbol{U}=$ $\operatorname{diag}\left\{\boldsymbol{U}_{0}, \boldsymbol{U}_{1}, \ldots, \boldsymbol{U}_{D-1}\right\}$, where $\boldsymbol{U}_{i}, i=0,1, \ldots, D-1$ is a $\left(K_{i} \times K_{i}\right)$ unitary matrix, which contains the $K_{i}$ orthonormal codes implementing the CDM of the $K_{i}$ symbols within the $i$ th group. Explicitly, we have $\sum_{i=0}^{D-1} K_{i}=K$. Let the indexes of the $K_{i}$ symbols falling within the $i$ th group be collected into a set $\mathcal{F}_{i}$. Then, these $D$ sets of indexes satisfy the constraints of $\bigcup_{i=0}^{D-1} \mathcal{F}_{i}=\{0,1, \ldots, K-1\}$ and $\mathcal{F}_{i} \cap \mathcal{F}_{j}=\varnothing, \forall i \neq j$, where $\varnothing$ denotes an empty set, whereas the symbol $\forall$ means "for all."

Note again that the GMC-CDM scheme includes both OFDM and MC-CDM schemes as its special examples. It can be seen that we have $K_{i}=1$ when $D=K$, implying that each group contains only one data symbol. In this case, we have $\boldsymbol{U}=$ $I_{K}$, and the GMC-CDM scheme reduces to an OFDM scheme. By contrast, we have $K_{i}=K$ when $D=1$, corresponding to the scenario that there is only one group containing all the $K$ data symbols. Correspondingly, the GMC-CDM scheme reduces to the MC-CDM scheme.

Furthermore, it is worthy of noting that, as shown in [17], the spreading matrix $\boldsymbol{P}$ is designed to satisfy the condition that each row of $\boldsymbol{P}$ contains only one nonzero entry to achieve low-complexity detection by employing one-tap post-FDE. 
Additionally, for the spreading to satisfy the power constraint, the spreading matrix is also required to satisfy $\sum_{m=0}^{M-1}\left|P_{m, j}\right|^{2}=1$ for $j=0,1, \ldots, K-1$, where $P_{m, j}$ denotes the $(m, j)$ th element of $\boldsymbol{P}$.

The GMC-CDM signal of (1) is transmitted over frequencyselective fading channels, after using inverse fast Fourier transform to transform the signal from the frequency domain to the time domain, cyclic prefixing, and pulse waveform shaping [9]. At the receiver side, after pulse waveform matched filtering, sampling, cyclic prefix removing, and the fast Fourier transform, it can be shown that the received signals from the $M$ subcarriers can be expressed as

$$
\boldsymbol{y}=\Lambda s+n=\Lambda W_{\mathrm{pre}} d+n
$$

where

y $M$-length observation vector containing the observations obtained from the $M$ subcarriers;

$\Lambda \quad(K \times K)$ diagonal matrix whose $m$ th diagonal entry, i.e., $\Lambda_{m}$, represents the channel response associated with the $m$ th subcarrier;

$n \quad M$-length noise vector, which is Gaussian distributed with zero mean and a covariance matrix $E\left[\boldsymbol{n} \boldsymbol{n}^{H}\right]=$ $\sigma_{n}^{2} \boldsymbol{I}_{M}$, where $\sigma_{n}^{2}=N_{0} / E_{s}$, and $E_{s} / N_{0}$ denotes the signal-to-noise ratio (SNR) per symbol.

Finally, the received signal of (2) is processed by a $(K \times M)$ postprocessing matrix $\boldsymbol{W}_{\text {post }}$, generating the decision variable vector

$$
\hat{\boldsymbol{d}}=\boldsymbol{W}_{\mathrm{post}} \boldsymbol{y}=\boldsymbol{W}_{\mathrm{post}} \boldsymbol{\Lambda} \boldsymbol{W}_{\mathrm{pre}} \boldsymbol{d}+\boldsymbol{W}_{\mathrm{post}} \boldsymbol{n}
$$

and based on this, the $K$ data symbols transmitted are detected.

\section{Post-Frequency Domain Equalizer}

To attain a good tradeoff between throughput and reliability, in this paper, we derive the optimum pre- and postprocessing matrices $\boldsymbol{W}_{\text {pre }}$ and $\boldsymbol{W}_{\text {post }}$, as shown in (3), jointly. Following many joint transceiver design methods, as illustrated, e.g., in [13] and [14], we first derive the optimum postprocessing matrix $\boldsymbol{W}_{\text {post }}$ when assuming that the preprocessing matrix $\boldsymbol{W}_{\text {pre }}$ is fixed. Then, for the given optimum postprocessing matrix $\boldsymbol{W}_{\text {post }}$, the optimum preprocessing matrix $\boldsymbol{W}_{\text {pre }}$ is derived.

The studies in [2] and [14] show that the detector based on the MMSE criterion is the optimum linear detector in terms of maximizing both throughput and reliability. Hence, we also adopt the MMSE criterion for postprocessing (detection). Then, based on (3) and (1), the optimum postprocessing matrix in MMSE sense can be derived, which is

$$
\begin{aligned}
\boldsymbol{W}_{\mathrm{post}} & =\left(\boldsymbol{W}_{\mathrm{pre}}^{H} \boldsymbol{\Lambda}^{H} \boldsymbol{\Lambda} \boldsymbol{W}_{\mathrm{pre}}+\sigma_{n}^{2} \boldsymbol{I}_{K}\right)^{-1} \boldsymbol{W}_{\mathrm{pre}}^{H} \boldsymbol{\Lambda}^{H} \\
& =\boldsymbol{U}^{H}(\boldsymbol{G}_{\mathrm{pre}}^{H} \underbrace{\boldsymbol{P}^{H} \boldsymbol{\Lambda}^{H} \boldsymbol{\Lambda} \boldsymbol{P}}_{\boldsymbol{\Pi}} \boldsymbol{G}_{\mathrm{pre}}+\sigma_{n}^{2} \boldsymbol{I}_{K})^{-1} \boldsymbol{G}_{\mathrm{pre}}^{H} \boldsymbol{P}^{H} \boldsymbol{\Lambda}^{H} \\
& =\boldsymbol{U}^{H} \boldsymbol{G}_{\mathrm{post}} \boldsymbol{P}^{H} \boldsymbol{\Lambda}^{H}
\end{aligned}
$$

where, by definition

$$
\boldsymbol{G}_{\mathrm{post}}=\left(\boldsymbol{G}_{\mathrm{pre}}^{H} \boldsymbol{\Pi} \boldsymbol{G}_{\mathrm{pre}}+\sigma_{n}^{2} \boldsymbol{I}_{K}\right)^{-1} \boldsymbol{G}_{\mathrm{pre}}^{H}
$$

which is a post-FDE matrix. From (4), we can see that the detector first carries out correlation operation by multiplying the received signal $\boldsymbol{y}$ with $\boldsymbol{P}^{H} \boldsymbol{\Lambda}^{H}$, where $\boldsymbol{\Lambda}^{H}$ matches the multicarrier channels, whereas $\boldsymbol{P}^{H}$ achieves despreading. Then, the detector carries out post-FDE by multiplying the correlation output with an FDE matrix $\boldsymbol{G}_{\text {post }}$ of (5). Finally, a matrix $\boldsymbol{U}^{H}$ is multiplied on the post-FDE's output to remove both FDM and CDM applied at the transmitter.

It can be shown that the matrix $\boldsymbol{\Pi}=\boldsymbol{P}^{H} \boldsymbol{\Lambda}^{H} \boldsymbol{\Lambda} \boldsymbol{P}$, as defined in (4), is still a diagonal matrix. This is because each row of the spreading matrix $\boldsymbol{P}$ has only one nonzero entry, and $\boldsymbol{\Lambda}$ is a diagonal matrix. Let $\Pi=\operatorname{diag}\left\{\Pi_{0}, \Pi_{1}, \ldots, \Pi_{K-1}\right\}$. Then, we can readily show that the post-FDE matrix $\boldsymbol{G}_{\text {post }}$ can be expressed in a simple form as

$$
\boldsymbol{G}_{\mathrm{post}}=\operatorname{diag}\left\{\frac{G_{\mathrm{pre}}^{(0) *}}{\Pi_{0}\left|G_{\mathrm{pre}}^{(0)}\right|^{2}+\sigma_{n}^{2}}, \ldots, \frac{G_{\mathrm{pre}}^{(K-1) *}}{\Pi_{K-1}\left|G_{\mathrm{pre}}^{(K-1)}\right|^{2}+\sigma_{n}^{2}}\right\} .
$$

Equation (6) shows that post-FDE is a one-tap equalizer that requires no matrix inversion. Therefore, the post-FDE in the GMC-CDM systems can be implemented with low complexity.

However, we can observe from (4) that the postprocessing matrix $\boldsymbol{W}_{\text {post }}$ optimized in MMSE sense is dependent on the preprocessing matrix $\boldsymbol{W}_{\text {pre }}$. Therefore, the optimal postprocessing matrix $\boldsymbol{W}_{\text {post }}$ cannot be directly obtained from (4) until the optimum preprocessing matrix $\boldsymbol{W}_{\text {pre }}$ is prepared. This implies that the transmitter and the receiver, or pre- and post-FDE, cannot be optimized independently but, instead, are optimized jointly.

As shown in Appendix A, after the one-tap post-FDE, the SINR for detection of the $k$ th symbol is given by

$$
\gamma_{k}=\frac{1}{\sigma_{n}^{2}}\left(\sum_{j=0}^{K-1} \frac{\left|U_{j, k}\right|^{2}}{\Pi_{j} \Omega_{j}+\sigma_{n}^{2}}\right)^{-1}-1
$$

where

$U_{j, k} \quad(j, k)$ th element of $\boldsymbol{U}$;

$\Pi_{j} \quad j$ th diagonal entry of $\Pi$. From the relationship of $\boldsymbol{\Pi}=\boldsymbol{P}^{H} \boldsymbol{\Lambda}^{H} \boldsymbol{\Lambda} \boldsymbol{P}$, we can obtain $\Pi_{j}=$ $\sum_{m=0}^{M-1}\left|P_{m, j}\right|^{2}\left|\Lambda_{m}\right|^{2}$

$\Omega_{j} \quad \Omega_{j}=\left|G_{\mathrm{pre}}^{(j)}\right|^{2}$, as defined, associated with (1).

In (7), the terms $\left\{U_{j, k}\right\},\left\{\Pi_{j}\right\}$, and $\left\{\Omega_{j}\right\}$ are all related to the transmitter preprocessing. Hence, the achievable SINR $\gamma_{k}$ of the GMC-CDM system is dependent on pre-FDE.

\section{TRANSMitTER OptIMIZATION}

In this section, we consider transmitter optimization under the assumption that the transmitter is capable of acquiring CSI. From (7), we can find that the SINR $\gamma_{k}$ is only related to the power responses of the multicarrier channels in $\Lambda$, the 
spreading matrix $\boldsymbol{P}$, and the pre-FDE matrix $\boldsymbol{G}_{\text {pre }}$ but is independent of their phase responses. This is because the MMSEassisted post-FDE shown in (4) is capable of eliminating the effect of the phases introduced by multicarrier channels and that by the transmitter preprocessing. Therefore, the transmitter preprocessing is only dependent on the power responses of the multicarrier channels. Consequently, our objective of transmitter optimization can be reduced to design such spreading matrix $\boldsymbol{P}$ and one-tap pre-FDE matrix $\boldsymbol{G}_{\text {pre }}$ that can match the power responses of the multicarrier channels to attain the maximum SDR or the highest reliability.

According to our analysis in Sections II and III, we can see from the SINR expression in (7) that the spreading matrix $\boldsymbol{P}$ depends only on the terms of $\left\{\Pi_{j}\right\}$. By contrast, the one-tap pre-FDE matrix $\boldsymbol{G}_{\text {pre }}$ relies only on the terms of $\left\{\Omega_{j}\right\}$. Hence, the optimization of the spreading matrix $\boldsymbol{P}$ and that of the one-tap pre-FDE matrix $\boldsymbol{G}_{\text {pre }}$ can be carried out separately. Therefore, in the sequel, we first consider the optimization of the spreading matrix $\boldsymbol{P}$.

\section{A. Design of the Spreading Matrix}

From (7), we can readily find out that, for any $j, k=$ $0,1, \ldots, K-1, \partial \gamma_{k} / \partial \Pi_{j} \geq 0$ is satisfied. Hence, the SINR $\gamma_{k}$ of (7) is an increasing function of $\Pi_{j}$, i.e., $\gamma_{k}$ increases with the increase of $\Pi_{j}$. Therefore, to achieve the maximum SINR, the spreading matrix $\boldsymbol{P}$ should be designed to make the terms of $\left\{\Pi_{j}\right\}$ maximum. Note that, as shown in (7), the optimization of $\boldsymbol{P}$ is independent of a specific index of $k$. Once we obtain a spreading matrix $\boldsymbol{P}$ that results in an optimum set of $\left\{\Pi_{j}\right\}$, the obtained $\boldsymbol{P}$ should then be optimal for all $K$ symbols.

In [17], we have considered the scenario where CSI is not available to the transmitter. In this case, the optimum transmission scheme is to spread the transmitted signal on multiple subcarriers to achieve the so-called open-loop repetition diversity [18]. Specifically, for a GMC-CDM system having an SF of $N$, let us assume that, after CDM and FDM, the $j$ th output is spread on the subcarriers indexed by $m_{0}, m_{1}, \ldots, m_{N-1}$. Let us assume that maximal ratio combining (MRC) is employed by the receiver. Then, it can be shown that the decision variable is linearly dependent on $\Pi_{j}=\sum_{n=0}^{N-1}\left|\Lambda_{m_{n}}\right|^{2} / N$, yielding a diversity order of $N$ [17]. Similarly, when the GMC-CDM system is operated under the CSIT mode, signals can also be conveyed on multiple subcarriers for achieving the closed-loop repetition diversity. In more detail, let us assume a GMC-CDM system having an SF of $N$. Let us also assume that, after $\mathrm{CDM}$ and FDM, the $j$ th output is transmitted on the subcarriers indexed by $m_{0}, m_{1}, \ldots, m_{N-1}$, with the aid of the transmitter preprocessing implementing transmitter MRC (TMRC) [2]. Then, it can be shown that the decision variable is also linearly related to $\Pi_{j}=\sum_{n=0}^{N-1}\left|\Lambda_{m_{n}}\right|^{2} / N$ [2], which illustrates that the diversity gain achieved by the TMRC scheme is the same as that achieved by the MRC receiver for the GMC-CDM systems without CSIT.

The foregoing analysis implies that, when the GMC-CDM system is operated under the CSIT mode, the TMRC scheme is not optimum, and there should exist the transmission schemes, which outperform the TMRC scheme. As shown in [18]-[20], the optimum transmission scheme in this case is in fact a transmit selection diversity (TSD) scheme, which conveys signals only on the best of the $N$ subcarriers. The reason for TSD to be optimum can be explained as follows. Since the GMCCDM system is operated under the CSIT mode, the transmitter hence employs the knowledge about $\left\{\left|\Lambda_{m_{0}}\right|^{2},\left|\Lambda_{m_{1}}\right|^{2}\right.$, $\left.\ldots,\left|\Lambda_{m_{N-1}}\right|^{2}\right\}$. Let the transmitter transmit the $j$ th output after CDM and FDM only on the subcarrier corresponding to the maximum of $\left\{\left|\Lambda_{m_{0}}\right|^{2},\left|\Lambda_{m_{1}}\right|^{2}, \ldots,\left|\Lambda_{m_{N-1}}\right|^{2}\right\}$. Then, it can be shown that the decision variable is linearly dependent on $\Pi_{j}=\max \left\{\left|\Lambda_{m_{n}}\right|^{2}\right\}$. Since $\max \left\{\left|\Lambda_{m_{n}}\right|^{2}\right\} \geq$ $\sum_{n=0}^{N-1}\left|\Lambda_{m_{n}}\right|^{2} / N$, we are hence assured that the TSD scheme outperforms the TMRC scheme. Note that, in contrast to the TMRC scheme, which can only achieve the diversity gain, the TSD scheme is capable of achieving both the diversity gain and the SNR gain. In other words, in addition to the same diversity order as the TMRC scheme, the TSD scheme is also capable of generating higher average SNR than the TMRC scheme, since $E\left[\max \left\{\left|\Lambda_{m_{n}}\right|^{2}\right\}\right] \geq$ $E\left[\sum_{n=0}^{N-1}\left|\Lambda_{m_{n}}\right|^{2} / N\right]=\sum_{n=0}^{N-1} E\left[\left|\Lambda_{m_{n}}\right|^{2}\right] / N$.

Based on the aforementioned analysis, it now becomes explicit that the spreading matrix $\boldsymbol{P}$ should be designed to achieve $\Pi_{j}=\max \left\{\left|\Lambda_{m_{n}}\right|^{2}\right\}$ by transmitting the $K$ outputs after CDM and FDM on the $K$ out of the $M$ subcarriers, which correspond to the $K$ largest values in $\left\{\left|\Lambda_{0}\right|^{2},\left|\Lambda_{1}\right|^{2}, \ldots,\left|\Lambda_{M-1}\right|^{2}\right\}$. Let the $K$ subcarriers corresponding to these $K$ largest values be indexed by $m_{j}, j=0,1, \ldots, K-1$. Then, the optimum spreading matrix $\boldsymbol{P}^{(o)}$ can be structured as

$$
\left|P_{m, j}^{(o)}\right|= \begin{cases}1, & m=m_{j}, j=0,1, \ldots, K-1 \\ 0, & \text { otherwise. }\end{cases}
$$

Note that the phase of $P_{m, j}^{(o)}$ can be any value, as we have previously discussed. However, to facilitate the channel estimation carried out at the receiver, it is desirable for the transmitter preprocessing to introduce only constant phases to the transmitted signals. In this case, the phases of the received signals only vary with the channels and, hence, may be estimated relatively easily.

Let us now consider the design of $\boldsymbol{G}_{\text {pre }}$, which accounts for power allocation.

\section{B. Pre-FDE}

Since the one-tap pre-FDE matrix $G_{\text {pre }}$ reflects the transmission power allocated to each of the $K$ data symbols, the design of pre-FDE is subject to the constraint $\sum_{j=0}^{K-1} \Omega_{j} \leq K$. The design of pre-FDE can be formulated by the constrained optimization problem

$$
\begin{array}{ll} 
& \max \left\{f\left(\gamma_{0}, \ldots, \gamma_{K-1}\right)\right\} \\
\text { s.t. } & \sum_{j=0}^{K-1} \Omega_{j} \leq K, \quad \Omega_{j} \geq 0, j=0,1, \ldots, K-1
\end{array}
$$

where the objective function $f\left(\gamma_{0}, \ldots, \gamma_{K-1}\right)$ is dependent on the specific objective of optimization, and s.t. means "subject to." Specifically, if our objective is to achieve 
maximum throughput, then the SDR of $R\left(\gamma_{0}, \ldots, \gamma_{K-1}\right)=$ $\sum_{k=0}^{K-1} \log \left(1+\gamma_{k}\right)$ is the objective function. By contrast, if improving the reliability of the GMC-CDM system is our motivation, then the objective function can be chosen as the average correct detection probability, which is expressed as $P_{c}\left(\gamma_{0}, \ldots, \gamma_{K-1}\right)=1-\sum_{k=0}^{K-1} P_{e}\left(\gamma_{k}\right) / K$, where $P_{e}\left(\gamma_{k}\right)$ is the error probability for detecting the $k$ th data symbol $d_{k}$ at a given SINR $\gamma_{k}$. However, it is usually very hard to find the solutions based on maximizing the correct detection probability or minimizing the error detection probability, due to the nonlinear relationship between $P_{e}\left(\gamma_{k}\right)$ and $\gamma_{k}$. Alternatively, the minimum SINR of $\gamma_{\min }=\min \left\{\gamma_{0}, \ldots, \gamma_{K-1}\right\}$ can be used as the objective function to be maximized. This is because the error probability of the GMC-CDM system is usually dominated by the data symbols having the minimum SINR. Hence, once the minimum SINR is maximized, the overall error probability of the GMC-CDM system is, in general, minimized.

As shown in Section II, in GMC-CDM systems, the $K$ symbols to be transmitted simultaneously are first divided into $D$ groups supported by FDM, and then, the symbols assigned to the same group are supported by CDM. There is no interference among the $D$ groups due to the property of FDM. However, the symbols within a group may interfere with each other, as a consequence that the orthogonality of the codes for CDM might be destroyed by the frequency-selective fading channels. Therefore, the design of the pre-FDE matrix $\boldsymbol{G}_{\text {pre }}$ needs to take into account of the symbols within a group, the groups, as well as the GP. Hence, in our pre-FDE design as described in the next section, the design process is divided into three stages: The firststage pre-FDE deals with the symbols within one group and the second-stage pre-FDE finds a good balance among the groups, whereas the last stage considers the GP. It can be shown that the second-stage pre-FDE is dependent on the results of the first-stage pre-FDE, whereas the GP is dependent on both the first-stage and the second-stage pre-FDE.

1) First-Stage Pre-FDE: Let us specifically consider the $i$ th group, where $i=0,1, \ldots, D-1$, which contains $K_{i}$ symbols multiplexed with the aid of $K_{i}$ orthonormal codes. Let us assume that the orthonormal codes for the CDM of the $i$ th group are normalized to satisfy $\left|U_{j, k}\right|^{2}=1 / K_{i}$, where $j \in \mathcal{F}_{i}$, and $k$ corresponds to the $k$ th symbol in the $i$ th group, as detailed in Section II. Then, remembering that there is no interference among the groups, the SINR for the symbols in the $i$ th group, which is stated as the SINR of the ith group and can be obtained from (7) as

$$
\gamma_{\mathcal{F}_{i}}=\frac{K_{i}}{\sigma_{n}^{2}}\left(\sum_{j \in \mathcal{F}_{i}} \frac{1}{\Pi_{j} \Omega_{j}+\sigma_{n}^{2}}\right)^{-1}-1
$$

which shows that all the symbols in the $i$ th group attain the same SINR. Now, the optimization problem is to find a set of values for $\left\{\Omega_{j}\right\}$, which maximize $\gamma_{\mathcal{F}_{i}}$.

Since both the SDR and reliability of the GMC-CDM system improve as the SINR increases, the objective of the first-stage pre-FDE is to maximize the SINR of each individual group, regardless of the specific optimization objective. As shown in (10), $\left(\sum_{j \in \mathcal{F}_{i}} 1 /\left(\Pi_{j} \Omega_{j}+\sigma_{n}^{2}\right)\right)^{-1}$ is the harmonic mean of
$\left\{\Pi_{j} \Omega_{j}+\sigma_{n}^{2}\right\}$ and is a concave function of $\left\{\Omega_{j}\right\}$. Hence, $\gamma_{\mathcal{F}_{i}}$ in (10) is a concave function of $\left\{\Omega_{j}\right\}$. Let the total power allocated to the $i$ th group be $\bar{\Omega}_{i}$, which is obtained from the secondstage pre-FDE, as will be detailed in Section IV-B2. Then, the convex optimization problem for the first-stage pre-FDE can be described as

$$
\left\{\Omega_{j}\right\}=\arg \max _{\left\{\Omega_{m}\right\}}\left\{\gamma_{\mathcal{F}_{i}}\right\} \quad \text { s.t. } \quad \sum_{j \in \mathcal{F}_{i}} \Omega_{j} \leq \bar{\Omega}_{i} .
$$

Explicitly, the SINR in (10) monotonically decreases with $\sum_{j \in \mathcal{F}_{i}} 1 /\left(\Pi_{j} \Omega_{j}+\sigma_{n}^{2}\right)$. Hence, the problem of (11) can be reduced to a simpler convex optimization problem associated with the objective function

$$
L\left(\left\{\Omega_{j}\right\}, \lambda_{i}\right)=\sum_{m \in \mathcal{F}_{i}} \frac{1}{\Pi_{m} \Omega_{m}+\sigma_{n}^{2}}-\lambda_{i}\left(\sum_{m \in \mathcal{F}_{i}} \Omega_{m}-\bar{\Omega}_{i}\right)
$$

where $\lambda_{i}$ is the Lagrange multiplier. Finally, upon solving the equations obtained from $\partial L\left(\left\{\Omega_{j}\right\}, \lambda_{i}\right) / \partial \Omega_{j}=0$, we can readily obtain the optimum solutions to $\left\{\Omega_{j}\right\}$, which are

$$
\Omega_{j}=\left(\frac{1}{\sqrt{\lambda_{i}}} \frac{1}{\sqrt{\Pi_{j}}}-\frac{1}{\Pi_{j} / \sigma_{n}^{2}}\right)^{+}, \quad j \in \mathcal{F}_{i}
$$

for $i=0,1, \ldots, D-1$, where $x^{+}=x$ if $x \geq 0$; otherwise, $x^{+}=0$ if $x<0$, whereas the constant $\lambda_{i}$ is chosen to satisfy the power constraint of $\sum_{j \in \mathcal{F}_{i}} \Omega_{j} \leq \bar{\Omega}_{i}$.

It can be seen that the power allocation based on (13) is similar to the classical water-filling algorithm [2], [15], which aims at achieving the channel capacity of multipleinput-multiple-output (MIMO) channels [21]. By contrast, our power-allocation algorithm of (13) aims to achieve the maximum SINR of a group of symbols. For convenience, we refer to our power-allocation algorithm as Water-filling-II, whereas the classical water-filling algorithm is referred to as Water-filling-I.

Let us assume that, in the $i$ th group, there are $\tilde{K}_{i}$ symbols assigned with nonzero power, and let $\tilde{\mathcal{F}}_{i}$ be the set containing the corresponding indexes of these symbols. Then, we have

$$
\sum_{j \in \tilde{\mathcal{F}}_{i}} \Omega_{j}=\bar{\Omega}_{i} .
$$

Upon substituting (13) into the preceding equation, the constant $\lambda_{i}$ can be derived, which is

$$
\frac{1}{\sqrt{\lambda_{i}}}=\left(\frac{\bar{\Omega}_{i}}{\tilde{K}_{i}}+\frac{1}{\tilde{K}_{i}} \sum_{j \in \tilde{\mathcal{F}}_{i}} \frac{1}{\Pi_{j} / \sigma_{n}^{2}}\right) \frac{1}{\psi_{i}}
$$

where, by definition, $\psi_{i}=\tilde{K}_{i}^{-1} \sum_{l \in \tilde{\mathcal{F}}_{i}} 1 / \sqrt{\Pi_{l}}$, and $1 / \psi_{i}$ denotes the harmonic mean of $\sqrt{\Pi_{j}}$. Furthermore, when substituting (15) into (13), it can be shown that the closed-form solutions to $\left\{\Omega_{j}\right\}$ of the $i$ th group can be expressed as

$$
\Omega_{j}= \begin{cases}\left(\frac{\bar{\Omega}_{i}}{\tilde{K}_{i}}+\frac{1}{\tilde{K}_{i}} \sum_{l \in \tilde{\mathcal{F}}_{i}} \frac{\sigma_{n}^{2}}{\Pi_{l}}\right) \frac{1}{\psi_{i} \sqrt{\Pi_{j}}}-\frac{\sigma_{n}^{2}}{\Pi_{j}}, & j \in \tilde{\mathcal{F}}_{i} \\ 0, & j \notin \tilde{\mathcal{F}}_{i} .\end{cases}
$$


Finally, the SINR of the symbols in the $i$ th group, which are assigned with nonzero power, can be obtained by substituting (16) into (10).

From (13), it can be shown that, for the Water-filling-II as aforementioned, all the symbols of the $i$ th group can be allocated with nonzero power, provided that $1 / \sigma_{n}^{2} \geq$ $\left(K_{i} / \bar{\Omega}_{i}\right)\left(\left(\psi_{i} / \Pi_{j}\right)-\left(1 / K_{i}\right) \sum_{l \in \mathcal{F}_{i}} 1 / \Pi_{l}\right)$ is satisfied for each of $j \in \mathcal{F}_{i}$. In this case, we have $K_{i}=\tilde{K}_{i}, \mathcal{F}_{i}=\tilde{\mathcal{F}}_{i}$, and the SINR of the $i$ th group is given by

$$
\gamma_{\mathcal{F}_{i}-A}=\left(\frac{\bar{\Omega}_{i}}{K_{i} \sigma_{n}^{2}}+\frac{1}{K_{i}} \sum_{j \in \mathcal{F}_{i}} \frac{1}{\Pi_{j}}\right) \psi_{i}^{-2}-1 .
$$

From (17), it can be seen that when the SNR of $1 / \sigma_{n}^{2}$ is sufficiently high, the SINR can be approximately expressed as

$$
\gamma_{\mathcal{F}_{i}-A} \approx \frac{\bar{\Omega}_{i}}{K_{i} \sigma_{n}^{2}} \psi_{i}^{-2}=\frac{\bar{\Omega}_{i}}{K_{i}}\left(\frac{1}{K_{i}} \sum_{j \in \mathcal{F}_{i}} \frac{1}{\sqrt{\Pi_{j} / \sigma_{n}^{2}}}\right)^{-2}
$$

which shows that the SINR attained by Water-filling-II is linearly proportional to the square of the harmonic mean of $\left\{\sqrt{\Pi_{j} / \sigma_{n}^{2}}\right\}$.

By contrast, for the open-loop GMC-CDM system where CSIT is not available, it can be shown that, for a sufficiently high SNR of $1 / \sigma_{n}^{2}$, the SINR can be denoted by

$$
\begin{aligned}
\gamma_{\mathcal{F}_{i}-B} & =\frac{1}{\sigma_{n}^{2}}\left[\frac{1}{K_{i}} \sum_{j \in \mathcal{F}_{i}}\left(\frac{\bar{\Omega}_{i}}{K_{i}} \Pi_{j}+\sigma_{n}^{2}\right)^{-1}\right]^{-1}-1 \\
& \approx \frac{\bar{\Omega}_{i}}{K_{i}}\left(\frac{1}{K_{i}} \sum_{j \in \mathcal{F}_{i}} \frac{1}{\Pi_{j} / \sigma_{n}^{2}}\right)^{-1}
\end{aligned}
$$

which shows that the SINR achievable is linearly proportional to the harmonic mean of $\left\{\Pi_{j} / \sigma_{n}^{2}\right\}$.

When $x_{l}$ 's are arbitrary positive numbers, we can readily prove that $\left(\sum_{l \in \mathcal{F}_{i}} x_{l} / K_{i}\right)^{2} \leq \sum_{l \in \mathcal{F}_{i}} x_{l}^{2} / K_{i}$, which is in fact a special case of the Chebychev's inequality [22]. Applying this property to (18) and (19), we attain $\gamma_{\mathcal{F}_{i}-B} \leq \gamma_{\mathcal{F}_{i}-A}$. Therefore, the first-stage pre-FDE is capable of increasing the average SINR, creating SINR gain. Let us now consider the secondstage pre-FDE, which is operated at the group level.

2) Second-Stage Pre-FDE: The second-stage pre-FDE optimizes the power allocated to the $D$ groups, which may be designed for maximizing either the throughput or the reliability of the GMC-CDM system. Specifically, when the design motivates to achieve a high throughput, the second-stage preFDE then maximizes the SDR under the power constraint of $\sum_{i=0}^{D-1} \bar{\Omega}_{i} \leq K$. In this case, given the SINRs in the form of (17) after the first-stage pre-FDE, the SDR can be expressed as

$$
R=\sum_{i=0}^{D-1} K_{i} \log \left(1+\gamma_{\mathcal{F}_{i}-A}\right)
$$

which is a concave function of $\left\{\bar{\Omega}_{i}\right\}$. Hence, maximizing $R$ under the linear constraint $\sum_{i=0}^{D-1} \bar{\Omega}_{i} \leq K$ is a convex opti- mization problem, and the optimal solutions to $\left\{\bar{\Omega}_{i}\right\}$ can be obtained by solving the optimization problem

$$
\begin{array}{ll}
\left\{\bar{\Omega}_{i}\right\}= & \arg \max _{\left\{\bar{\Omega}_{l}\right\}}\left\{R=\sum_{i=0}^{D-1} K_{i} \log \left(1+\gamma_{\mathcal{F}_{i}-A}\right)\right\} \\
\text { s.t. } & \sum_{i=0}^{D-1} \bar{\Omega}_{i} \leq K .
\end{array}
$$

After solving this problem, we obtain

$$
\bar{\Omega}_{i}= \begin{cases}\frac{K_{i} \tilde{\xi}}{\frac{1}{K} \sum_{l \in \mathcal{S}} K_{l}}-\sum_{j \in \mathcal{F}_{i}} \frac{1}{\Pi_{j} / \sigma_{n}^{2}}, & i \in \mathcal{S} \\ 0, & i \notin \mathcal{S}\end{cases}
$$

where $\tilde{\xi}=1+(1 / K) \sum_{l \in \mathcal{S}} \sum_{j \in \mathcal{F}_{i}}\left(\Pi_{j} / \sigma_{n}^{2}\right)^{-1}$, and the set $\mathcal{S}$ contains the indexes of the groups allocated with nonzero power. Finally, when applying (17) associated with (22) into (20), the SDR achieved is given by

$$
R=\sum_{i \in \mathcal{S}} K_{i} \log \left(\frac{\tilde{\xi}}{\frac{\sigma_{n}^{2}}{K} \sum_{l \in \mathcal{S}} K_{l}}\right)-\log \left(\prod_{i \in \mathcal{S}} \psi_{i}^{2 K_{i}}\right) .
$$

By contrast, when the objective of optimization is to enhance the reliability of the GMC-CDM system, then, as aforementioned in this section, the objective function to be maximized can be set as $\gamma_{\text {min }}=\min \left\{\gamma_{\mathcal{F}_{0}-A}, \ldots, \gamma_{\mathcal{F}_{D-1}-A}\right\}$. However, solving this optimization problem is not straightforward, since the expression for $\gamma_{\min }$ is not explicit. Instead, this optimization problem can be solved by the approach detailed in Appendix B. As shown in Appendix B, the optimization results in that the power must be allocated, and therefore, the SINRs of all the groups are the same. Specifically, after substituting the terms from (39) into (48), we obtain the optimum solutions of the second-stage pre-FDE, which are

$$
\bar{\Omega}_{i}=\frac{\xi K_{i} \psi_{i}^{2}}{\frac{1}{K} \sum_{l=0}^{D-1} K_{l} \psi_{l}^{2}}-\sum_{j \in \mathcal{F}_{i}} \frac{1}{\Pi_{j} / \sigma_{n}^{2}}
$$

where $\xi=1+(1 / K) \sum_{l=0}^{D-1} \sum_{j \in \mathcal{F}_{l}}\left(\Pi_{j} / \sigma_{n}^{2}\right)^{-1}$. Furthermore, upon substituting the terms from (39) into (49), it can be shown that

$$
\gamma_{\mathcal{F}_{0}-A}=\cdots=\gamma_{\mathcal{F}_{D-1}-A}=\gamma_{c}=\frac{\xi}{\frac{\sigma_{n}^{2}}{K} \sum_{i=0}^{D-1} K_{i} \psi_{i}^{2}}-1 .
$$

Note that, in (22), $\tilde{\xi}$ is constant, whereas the reliability of the $i$ th group provided by the first-stage pre-FDE is inversely dependent on $\sum_{j \in \mathcal{F}_{i}}\left(\sigma_{n}^{2} / \Pi_{j}\right)$. Hence, when the second-stage pre-FDE motivates the achievement of the maximum throughput, the transmitter of the GMC-CDM system then assigns more power to the groups with relatively high reliabilities but less or even no power to the groups with relatively low reliabilities. By contrast, for the second-stage pre-FDE aiming at improving the reliability of the GMC-CDM system, if the SNR is sufficiently high, i.e., if $\sigma_{n}^{2}$ is sufficiently low, (24) can be approximated by

$$
\bar{\Omega}_{i} \approx \frac{\xi K_{i} \psi_{i}^{2}}{\frac{1}{K} \sum_{l=0}^{D-1} K_{l} \psi_{l}^{2}}
$$


where $\xi$ and the denominator are constants, whereas $\psi_{i}^{2}$ is inversely dependent on the reliability of the $i$ th group provided by the first-stage pre-FDE. Hence, when the second-stage preFDE motivates to improve the reliability of the GMC-CDM system, more power is then assigned to the groups having relatively low reliabilities, whereas less power is assigned to the groups with relatively high reliabilities so that all the groups are capable of reaching the same SINR, as shown in (25), after the second-stage pre-FDE.

Finally, when considering both the first-stage and the secondstage pre-FDE, the power allocation for maximizing throughput (or SDR) can be obtained by combining (22) with (16), yielding

$$
\Omega_{j}=\frac{\tilde{\xi}}{\frac{1}{K} \sum_{l \in \mathcal{S}} K_{l}} \frac{1}{\psi_{i} \sqrt{\Pi_{j}}}-\frac{1}{\Pi_{j} / \sigma_{n}^{2}}, \quad j \in \mathcal{F}_{i} .
$$

The power allocation for achieving high reliability can be obtained by substituting (24) into (16), which results in

$$
\Omega_{j}=\frac{\xi}{\frac{1}{K} \sum_{l=0}^{D-1} K_{l} \psi_{l}^{2}} \frac{\psi_{i}}{\sqrt{\Pi_{j}}}-\frac{1}{\Pi_{j} / \sigma_{n}^{2}}, \quad j \in \mathcal{F}_{i} .
$$

Note that, as aforementioned, the GMC-CDM system is reduced to a MC-CDM system when $D=1$. In this case, there is no second-stage pre-FDE, and both (27) and (28) become the same, representing Water-filling-II as discussed in Section IV-B1. By contrast, the GMC-CDM system is reduced to a conventional OFDM system when $D=K$. In this case, no first-stage pre-FDE is necessary. Correspondingly, the power allocation of (27) represents the conventional water filling, defined as Water-filling-I in Section IV-B1, whereas the power allocation of (28) denotes a power-control scheme based on the channel inversion principles [15].

3) GP: The requirement for the GP can be inferred by (23) and (25). As shown in (23) and (25), both the achievable SDR and achievable SINR are dependent on $\psi_{i}=$ $K_{i}^{-1} \sum_{l \in \mathcal{F}_{i}} 1 / \sqrt{\Pi_{l}}$, which, explicitly, is related to the GP. Hence, the achievable SDR and SINR are dependent on the GP, which assigns the $K$ data symbols into $D$ groups. In this paper, for the sake of simplicity, we assume that all the $D$ groups contain the same number of data symbols, implying that $K_{i}=K / D$ for $i=0,1, \ldots, D-1$. Let $K_{i}=K / D$ in (23). Then, the second term in (23) can be expressed as $-2 K \log \left(\sqrt[D]{\prod_{i=0}^{D-1} \psi_{i}}\right)$, showing that the SDR $R$ monotonically decreases as the geometric mean (GM) of $\psi_{i}$ increases. Similarly, let $K_{i}=K / D$ in (25); then, the denominator of the first term in (25) can be expressed as $\sigma_{n}^{2}\left(\sqrt{D^{-1} \sum_{i=0}^{D-1} \psi_{i}^{2}}\right)^{2}$, explaining that the achievable SINR of $\gamma_{c}$ monotonically decreases as the root mean square (RMS) of $\psi_{i}$ increases. Therefore, given $D$ groups with each group handling $K / D$ symbols, the GP can be described by the optimization problem

$$
\left\{\mathcal{F}_{i}\right\}=\arg \min _{\left\{\mathcal{F}_{l}\right\}} \sqrt[D]{\prod_{l=0}^{D-1}\left(\frac{D}{K} \sum_{j \in \mathcal{F}_{l}} \frac{1}{\sqrt{\Pi_{j}}}\right)}
$$

when maximizing the SDR and by the optimization problem

$$
\left\{\mathcal{F}_{i}\right\}=\arg \min _{\left\{\mathcal{F}_{l}\right\}} \sqrt{\frac{1}{D} \sum_{l=0}^{D-1}\left(\frac{D}{K} \sum_{j \in \mathcal{F}_{l}} \frac{1}{\sqrt{\Pi_{j}}}\right)^{2}}
$$

when maximizing the SINR. In both (29) and (30), $\psi_{i}=$ $K_{i}^{-1} \sum_{l \in \mathcal{F}_{i}} 1 / \sqrt{\Pi_{l}}$ associated with $K_{i}=K / D$ has been applied.

It can be shown that the aforementioned two optimization problems belong to the class of combinational optimization problems that are NP-hard. However, it is well known that the GM of $\left\{\psi_{i}\right\}$ has the property that it reduces when the difference of $\left\{\psi_{i}\right\}$ between any two groups increases. By contrast, the RMS of $\left\{\psi_{i}\right\}$ decreases when the difference of $\left\{\psi_{i}\right\}$ between any two groups decreases. For this sake, we propose heuristic solutions to the GP optimization problem, which are described in the list that follows.

- When the design aims to achieve a high throughput, then, given $K_{i}=K / D, i=0,1, \ldots, D-1$, the symbols to be transmitted on the subcarriers experiencing similar fading are assigned to the same group. In other words, the symbols corresponding to the $K_{0}$ largest values in $\left\{\Pi_{0}, \ldots, \Pi_{K-1}\right\}$ are assigned to the first group, the symbols corresponding to the next $K_{1}$ largest values in $\left\{\Pi_{0}, \ldots, \Pi_{K-1}\right\}$ are assigned to the second group, and so on until the last $K_{D-1}$ symbols are assigned to the $(D-1)$ th group. As a result of this GP, the difference of $\left\{\psi_{i}\right\}$ between any two groups is maximized, and hence, the SDR of (23) can be maximized.

- When the design aims to achieve a high reliability, then, given $K_{i}=K / D, i=0,1, \ldots, D-1$, the symbols to be transmitted on the subcarriers experiencing different fading, yielding different reliabilities, should be assigned to the same group. For example, we can assign the symbols to be transmitted by the best and worst subcarriers to the same group. By doing this type of GP, all the $D$ groups will have a similar value for $\left\{\psi_{i}\right\}$, making the difference of $\left\{\psi_{i}\right\}$ between any two groups as small as possible and, ultimately, making the minimum SINR of (25) maximum.

As aforementioned, we have considered the optimization of pre-FDE, which consists of three stages of optimization, including the first-stage and the second-stage pre-FDE, as well as the last stage of the GP. In summary, the joint transmitter-receiver optimization can be implemented as follows. First, the GP is carried out to assign the $K$ symbols into different groups expressed as $\left\{\mathcal{F}_{i}\right\}$, based on the objective of maximum SDR or maximum reliability. Second, depending on the optimization objective of maximum SDR or maximum reliability, power allocation is executed according to (22) or (24) within each of the groups, obtaining $\left\{\bar{\Omega}_{i}\right\}$. Third, given $\left\{\bar{\Omega}_{i}\right\}$, the optimum power responses of $\left\{\Omega_{j}\right\}$ for the $K$ symbols are calculated using (16). Additionally, the spreading matrix is designed based on (8). Finally, the postprocessing matrix achieving MMSE detection can be obtained from (4). 


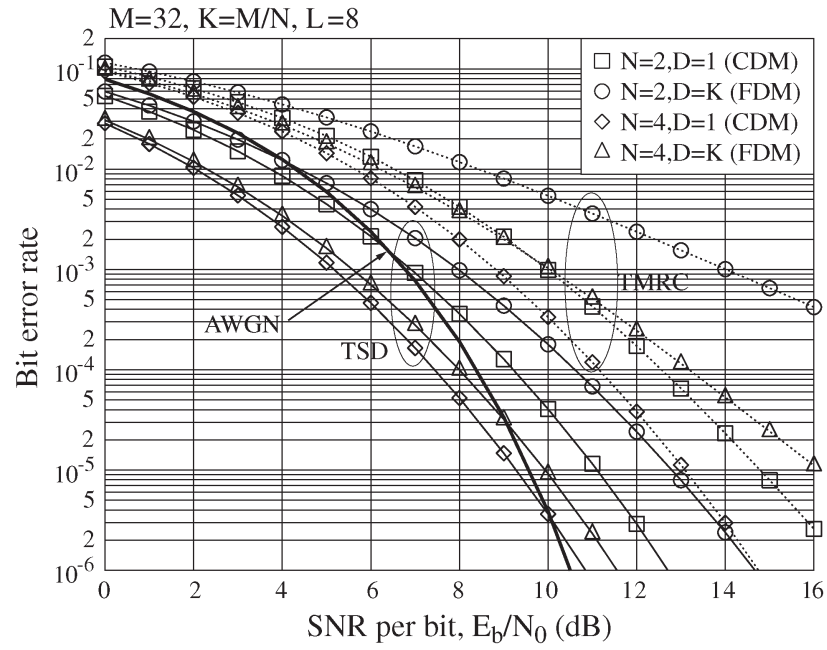

Fig. 1. BER versus SNR per bit performance comparison of the GMCCDM systems employing the transmit diversity based on TMRC (i.e., transmit repetition diversity) or TSD.

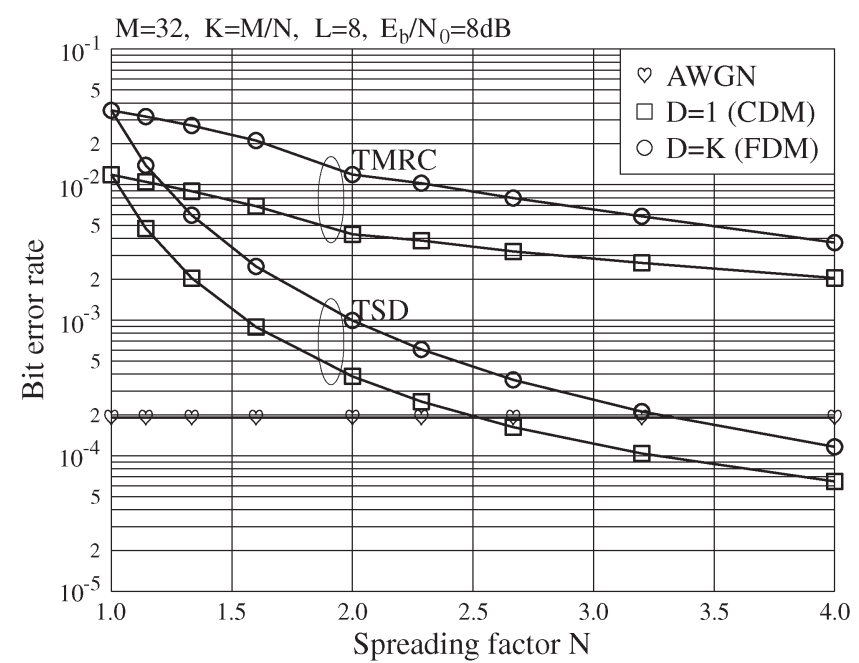

Fig. 2. BER versus spreading factor $N$ performance for the GMC-CDM systems employing the transmit diversity based on TMRC or TSD.

\section{PERFormance Results}

In this section, we illustrate a range of performance results for characterizing the achievable performance of GMC-CDM systems employing $M=32$ subcarriers. In our simulations, we assumed that the GMC-CDM signals were transmitted over frequency-selective Rayleigh fading channels having $L$ time-domain resolvable paths. Furthermore, for the examples considering reliability, we assumed that quadrature phase-shift keying (QPSK) modulation was employed. By contrast, when the optimization objective was maximum SDR, we assumed the ideal adaptive modulation and/or coding schemes, which could be implemented for attaining any data rate. Note that the corresponding parameters used in our simulations are displayed in association with the figures.

Figs. 1 and 2 compare the bit error rate (BER) of the GMC-CDM systems using TMRC- or TSD-assisted transmit diversity, as discussed in Section IV-A. As shown in Figs. 1 and 2, for any given parameters of $N$ and $D$, the TSDbased transmit diversity scheme significantly outperforms the

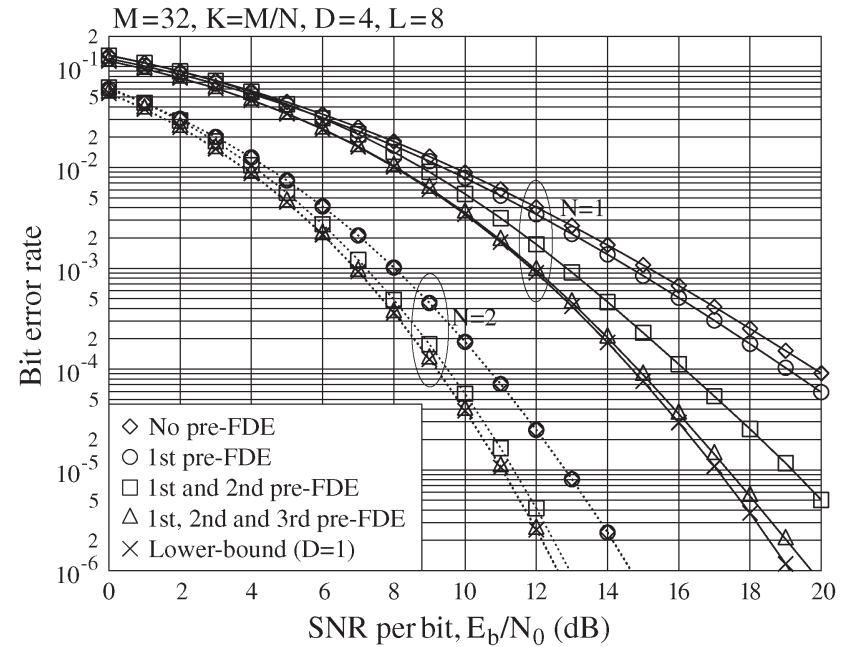

Fig. 3. BER versus SNR per bit performance for the GMC-CDM systems using different levels of pre-FDE.

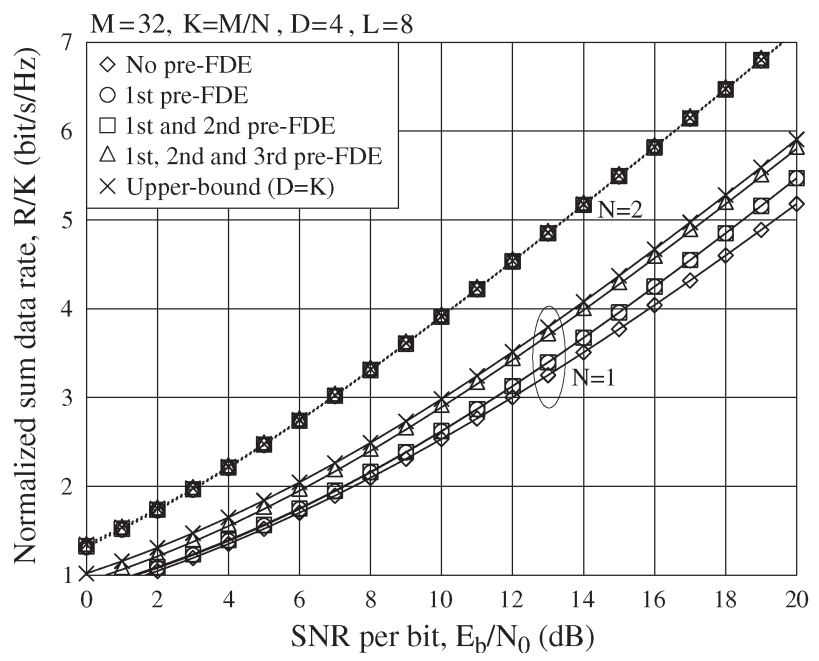

Fig. 4. SDR versus SNR per bit performance of the GMC-CDM systems using different levels of pre-FDE.

TMRC-based transmit diversity scheme. Furthermore, Fig. 1 shows that if the SNR $E_{b} / N_{0}$ is low, the TSD-based transmit diversity scheme is even capable of achieving better BER than that attained over additive white Gaussian noise channels. This is also observed in Fig. 2, when the SF $N$ is sufficiently high. As discussed in Section II, the GMC-CDM system is reduced to the MC-CDM system when $D=1$ and to the FDM system when $D=K$. Since the MC-CDM scheme can attain frequency diversity but the FDM scheme cannot, as shown in Figs. 1 and 2, the BER for the cases of $D=1$ is much better than that for the corresponding cases of $D=K$.

Figs. 3 and 4 illustrate the impact of the three stages of pre-FDE on the achievable BER (see Fig. 3) or SDR (see Fig. 4) performance of the GMC-CDM systems, which employ $M=32$ subcarriers and the $\mathrm{SF}$ of $N=1$ or 2 . Furthermore, in Fig. 3, the BER lower bound achieved by the MC-CDM system corresponding to $D=1$ is depicted, whereas in Fig. 4, the SDR upper bound is depicted, for the sake of comparison. The results of Fig. 3 show that, when $N=1$, employing preFDE can significantly improve the BER of the GMC-CDM 


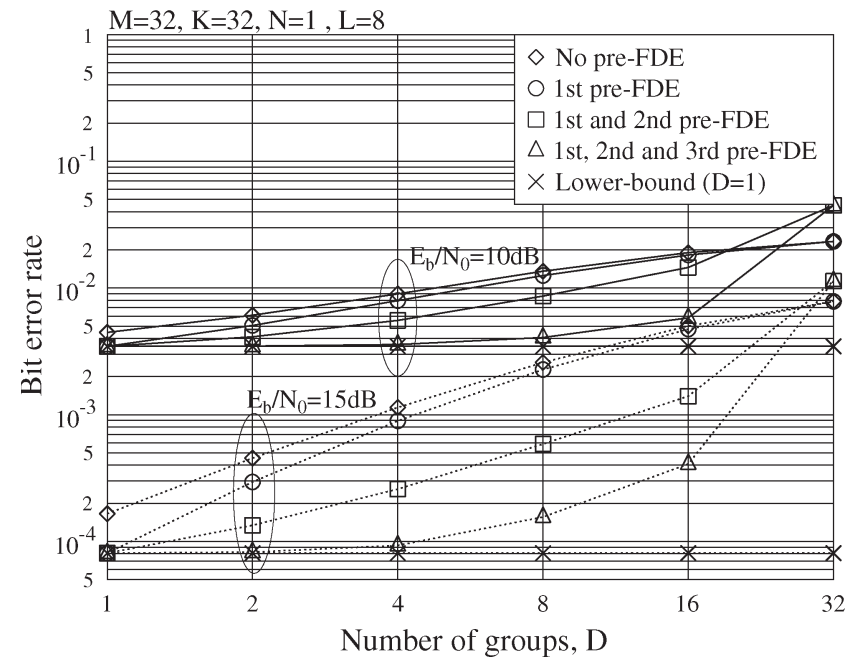

Fig. 5. BER versus the number of groups used by the GMC-CDM systems associated with using different levels of pre-FDE.

systems, in comparison with the GMC-CDM systems without using pre-FDE. Specifically, using only the first-stage pre-FDE slightly enhances the BER. The second-stage pre-FDE adds a big improvement to the achievable BER. The third-stage preFDE, i.e., the GP, also makes a big contribution to the BER improvement. Furthermore, due to the diversity gain attained by the three-stage pre-FDE, we can observe that the BER improvement becomes more explicit as the SNR increases. As shown in Fig. 3, when $N=2$, the BER improvement is not as significant as that for the cases of $N=1$, but about $2 \mathrm{~dB}$ of SNR gain can still be achievable at the BER of $10^{-6}$. Additionally, Fig. 3 shows that, with the aid of pre-FDE, the BER achieved by a GMC-CDM system is close to the BER lower bound achieved by the corresponding MC-CDM system.

With regard to the SDR performance, from the results of Fig. 4, we can observe that, for $N=1$ and at a given SNR per bit value, the GMC-CDM scheme without using pre-FDE achieves the lowest SDR. The highest SDR is achieved by the FDM scheme corresponding to $D=K=32$ associated with using ideal water filling for power allocation. When pre-FDE and GP are employed, the GMC-CDM scheme with $D=4$ is capable of achieving the SDR that is close to that achieved by the FDM scheme using ideal water filling. However, it is worth noting that the FDM system can only attain the BER as the QPSK scheme communicating over flat Rayleigh fading channels, which is much worse than that of the GMC-CDM system using $K=32, D=4$, and $N=1$, as observed in Fig. 3. Furthermore, the results of Fig. 4 corresponding to $N=1$ show that the proposed GP is also capable of increasing the SDR of the GMC-CDM systems. By contrast, for the case of $N=2$, as shown in Fig. 4, using pre-FDE does not result in further improvement of SDR, in comparison with the GMC$\mathrm{CDM}$ using solely post-FDE. The reason for this observation is that the GMC-CDM system is half-loaded, i.e., $K / M=1 / 2$, when $N=2$. It is a typical under-load MIMO system with $K$ inputs and $M=2 K$ outputs, whose capacity can be achieved without requiring CSI known to the transmitter [2], [23].

Figs. 5 and 6 illustrate the effect of the number of groups on the achievable BER and SDR performance. In general, for

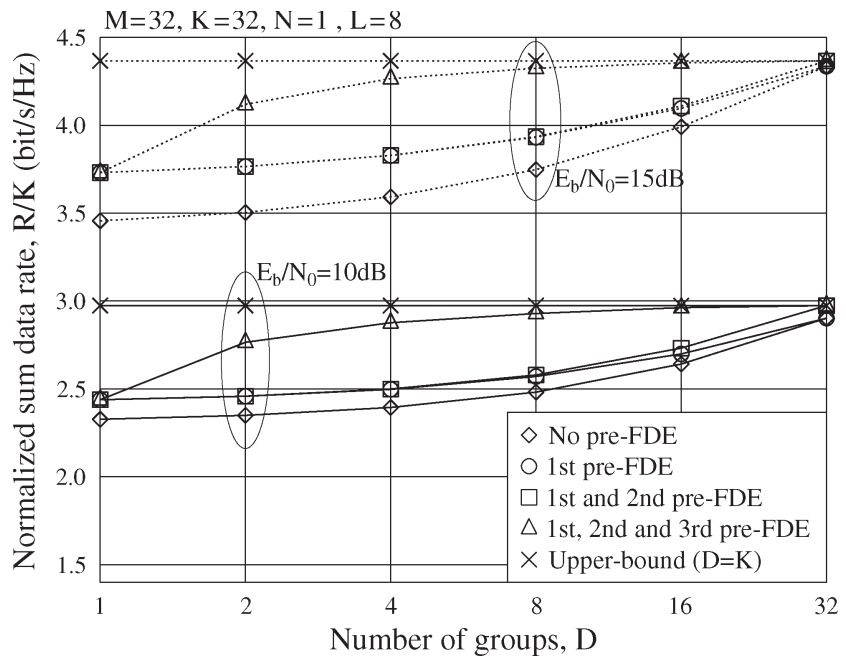

Fig. 6. SDR versus the number of groups used by the GMC-CDM systems associated with using different levels of pre-FDE.

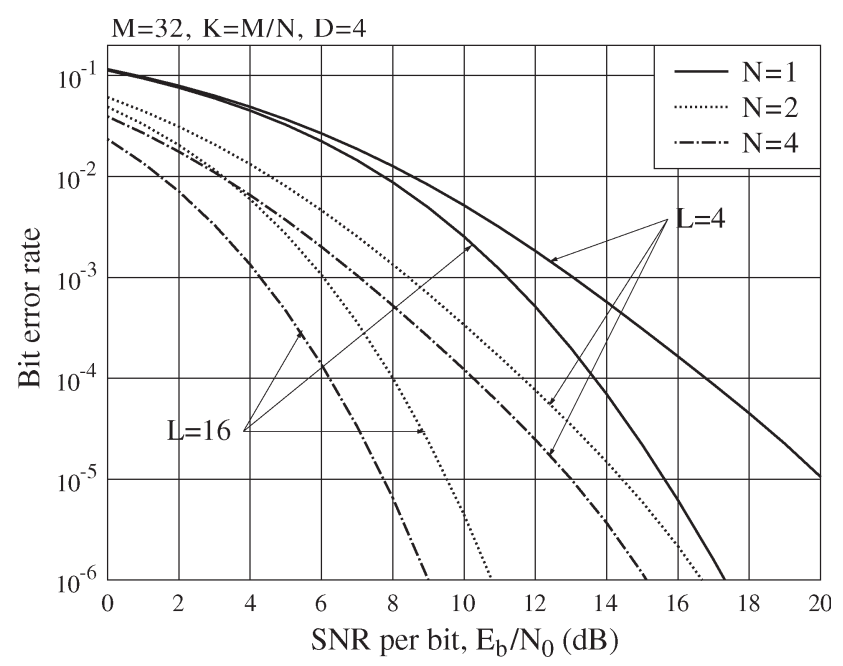

Fig. 7. Effect of the number of resolvable multipaths of wireless channels on the BER versus SNR per bit performance of the GMC-CDM systems using both pre- and post-FDE.

a given SNR of $E_{b} / N_{0}$ and a given pre-FDE strategy, the BER becomes worse while the SDR improves, as the number of groups increases. This observation explains that, given a throughput required, there exists an optimum value for $D$ of the number of groups, which results in the lowest BER or highest reliability. Additionally, from Fig. 6, we can see that, when employing the GP (curves marked with triangles), the SDR significantly increases when the number of groups increases from $D=1$ to $D=2$ and to $D=4$ and then insignificantly increases when further increasing the number of groups. Inversely, Fig. 5 shows that when employing the GP (curves also marked with triangles), the BER is nearly the same when the number of groups used is $D=1,2$, or 4 . However, the BER becomes very sensitive to the number of groups, as it is increased beyond $D=4$. The preceding observation implies that, in a GMC-CDM system, it is desirable to use a relative low number of groups. By doing this, most of the SDR can be attained with a low loss of BER. 


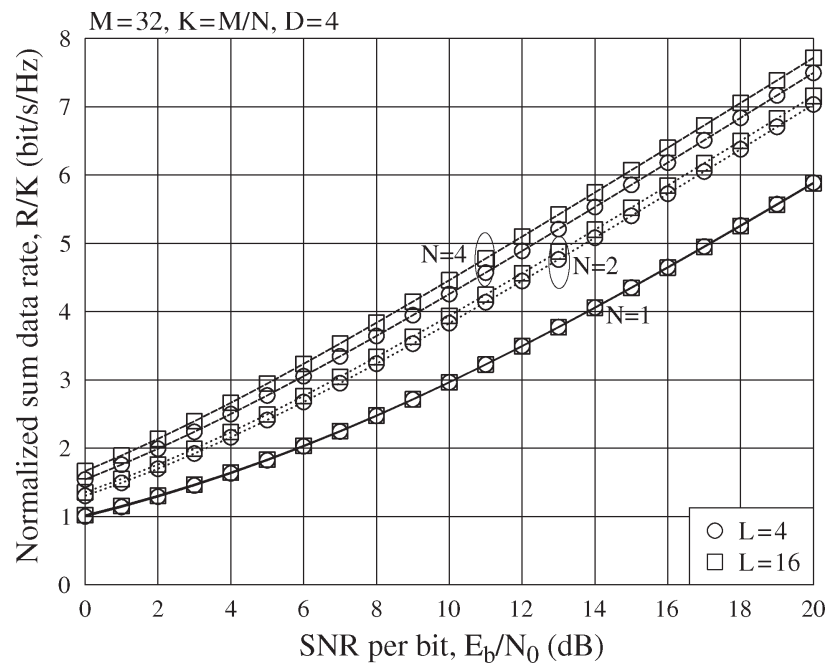

Fig. 8. Effect of the number of resolvable multipaths of wireless channels on the BER versus SNR per bit performance of the GMC-CDM systems using both pre- and post-FDE.

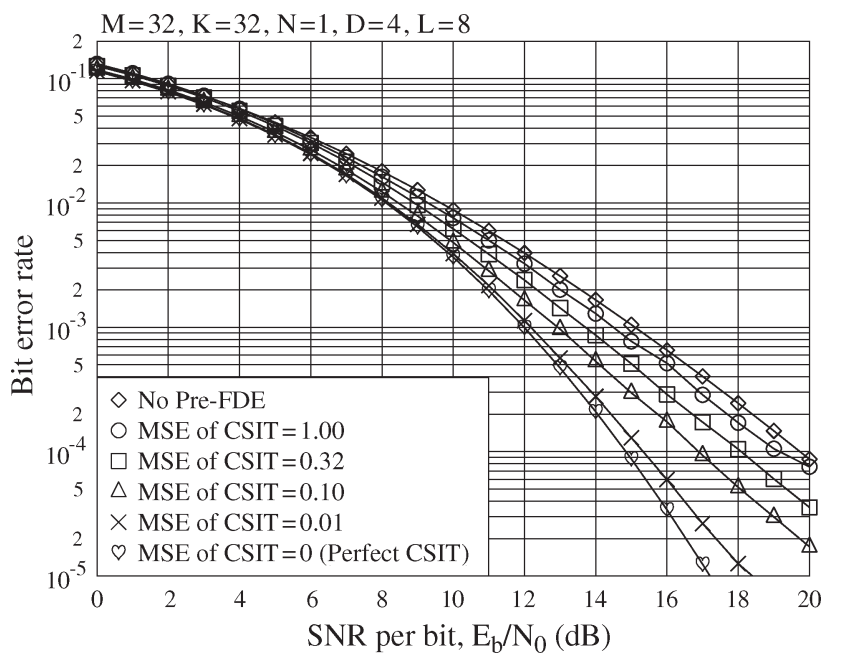

Fig. 9. Effect of the outdated CSIT on the BER versus SNR per bit performance of the GMC-CDM systems.

In Figs. 7 and 8, we investigate the impact of the frequency-selective channels on the achievable BER and SDR performance. Explicitly, the BER improves as the number of resolvable multipaths increases, i.e., as the fading of wireless channels becomes more frequency selective. For a given SF $N$, the SDR slightly increases as the fading becomes more frequency selective. This observation is more declared when the value of $N$ becomes higher.

In the aforementioned figures, the performance results of the GMC-CDM systems were obtained under the assumption that the transmitter was capable of acquiring ideal CSI. In practice, however, the transmitter may have to use outdated CSI for preprocessing, owing to the time-varying characteristics of wireless channels [24]. Therefore, in Figs. 9 and 10, we investigate the impact of the outdated CSIT on the achievable BER and SDR performance of the MC-CDM systems. Owing to the joint transmitter-receiver optimization, as shown in Figs. 9 and 10, the SDR or BER performance of the MC-CDM systems using pre-FDE will never become worse than that of the MC-CDM systems using no pre-FDE, no matter what the mean

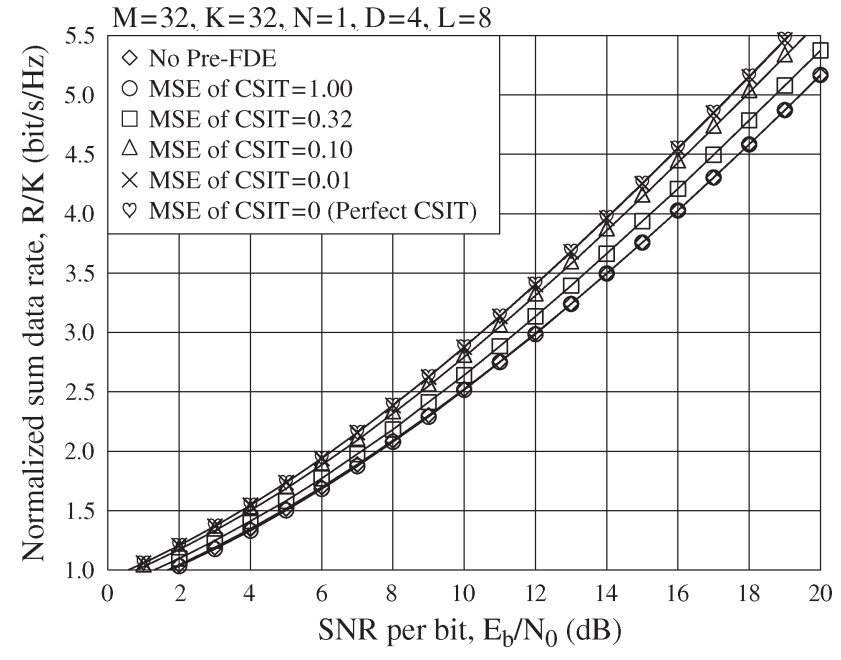

Fig. 10. Effect of the outdated CSIT on the SDR versus SNR per bit performance of the GMC-CDM systems.

square error $\left(\mathrm{MSE}^{1}\right)$ of CSIT is. The SDR or BER performance of the GMC-CDM systems improves as the MSE of CSIT decreases, which approaches the SDR or BER performance of the GMC-CDM systems with perfect CSIT, when the MSE of CSIT becomes less than 0.01 .

\section{CONCLUSION}

Joint transmitter-receiver optimization for GMC-CDM systems has been investigated. Both the low-complexity pre- and post-FDE algorithms have been derived, which can significantly improve the reliability or throughput of the GMC-CDM systems. In the conventional multicarrier schemes, such as MC-CDMA and OFDM, there usually exists an explicit tradeoff between BER and throughput, either the best BER and the lowest throughput, or vice versa. However, by dividing the symbols to be transmitted into a relatively low number of groups and carrying out the corresponding pre- and post-FDE, our GMC-CDM system is generally capable of achieving most of the available SDR but with a very low loss of BER. In essence, the proposed pre- and post-FDE algorithms facilitate the GMC-CDM systems to make efficient use of the power and frequency resources. Furthermore, these pre- and post-FDE algorithms can be operated with high flexibility to attain a good tradeoff between throughput and reliability.

\section{APPENDIX A}

DERIVATION OF (7)

In this Appendix, we derive the expression of (7) based on (3). We start with expressing the decision variable of the $k$ th symbol as

$$
\hat{d}_{k}=\boldsymbol{e}_{k}^{H}\left(\boldsymbol{U}^{H} \boldsymbol{G}_{\text {post }} \boldsymbol{\Pi} \boldsymbol{G}_{\text {pre }} \boldsymbol{U} \boldsymbol{d}+\tilde{\boldsymbol{n}}\right)
$$

where $e_{k}$ is a basis vector with the $k$ th entry being one while the other entries are zeros, and as shown in Section III, $\tilde{\boldsymbol{n}}$ is Gaussian distributed associated with zero mean and a covariance matrix $E\left[\tilde{\boldsymbol{n}} \tilde{\boldsymbol{n}}^{H}\right]=\sigma_{n}^{2} \boldsymbol{U}^{H} \boldsymbol{G}_{\mathrm{post}} \boldsymbol{\Pi} \boldsymbol{G}_{\text {post }}^{H} \boldsymbol{U}$.

\footnotetext{
${ }^{1}$ Note that to highlight the impact of time-varying wireless channels, the MSE of CSIT considered in our simulations is only led by the outdated CSIT. The channel-estimation errors due to noise or interference are not considered.
} 
From (31), one can know that the signal power is

$$
\left|E\left[\hat{d}_{k}\right]\right|^{2}=\left(\boldsymbol{e}_{k}^{H} \boldsymbol{U}^{H} \boldsymbol{G}_{\mathrm{post}} \boldsymbol{\Pi} \boldsymbol{G}_{\mathrm{pre}} \boldsymbol{U} \boldsymbol{e}_{k}\right)^{2} .
$$

The interference-plus-noise power is

$$
\begin{aligned}
\sigma_{k}^{2}= & E\left[\left|\hat{d}_{k}\right|^{2}\right]-\left|E\left[\hat{d}_{k}\right]\right|^{2} \\
= & \boldsymbol{e}_{k}^{H}\left(\boldsymbol{U}^{H} \boldsymbol{G}_{\mathrm{post}} \boldsymbol{\Pi} \boldsymbol{G}_{\mathrm{pre}} \boldsymbol{U} \boldsymbol{U}^{H} \boldsymbol{G}_{\mathrm{pre}}^{H} \boldsymbol{\Pi} \boldsymbol{G}_{\mathrm{post}}^{H} \boldsymbol{U}\right. \\
& \left.\quad+\sigma_{n}^{2} \boldsymbol{U}^{H} \boldsymbol{G}_{\mathrm{post}} \boldsymbol{\Pi} \boldsymbol{G}_{\mathrm{post}}^{H} \boldsymbol{U}\right) \boldsymbol{e}_{k} \\
& -\left(\boldsymbol{e}_{k}^{H} \boldsymbol{U}^{H} \boldsymbol{G}_{\mathrm{post}} \boldsymbol{\Pi} \boldsymbol{G}_{\mathrm{pre}} \boldsymbol{U} \boldsymbol{e}_{k}\right)^{2} \\
= & \boldsymbol{e}_{k}^{H} \boldsymbol{U}^{H} \boldsymbol{G}_{\mathrm{post}} \boldsymbol{\Pi}\left(\boldsymbol{G}_{\mathrm{pre}} \boldsymbol{G}_{\mathrm{pre}}^{H} \boldsymbol{\Pi}+\sigma_{n}^{2} \boldsymbol{I}_{K}\right) \boldsymbol{G}_{\mathrm{post}}^{H} \boldsymbol{U} \boldsymbol{e}_{k} \\
& -\left(\boldsymbol{e}_{k}^{H} \boldsymbol{U}^{H} \boldsymbol{G}_{\mathrm{post}} \boldsymbol{\Pi} \boldsymbol{G}_{\mathrm{pre}} \boldsymbol{U} \boldsymbol{e}_{k}\right)^{2} .
\end{aligned}
$$

Upon invoking (6), the preceding equation can be simplified to

$$
\begin{aligned}
\sigma_{k}^{2}= & \boldsymbol{e}_{k}^{H} \boldsymbol{U}^{H} \boldsymbol{G}_{\mathrm{post}} \boldsymbol{\Pi} \boldsymbol{G}_{\mathrm{pre}} \boldsymbol{U} \boldsymbol{e}_{k}-\left(\boldsymbol{e}_{k}^{H} \boldsymbol{U}^{H} \boldsymbol{G}_{\mathrm{post}} \boldsymbol{\Pi} \boldsymbol{G}_{\mathrm{pre}} \boldsymbol{U} \boldsymbol{e}_{k}\right)^{2} \\
= & \boldsymbol{e}_{k}^{H} \boldsymbol{U}^{H} \boldsymbol{G}_{\mathrm{post}} \boldsymbol{\Pi} \boldsymbol{G}_{\mathrm{pre}} \boldsymbol{U} \boldsymbol{e}_{k} \\
& \times\left(1-\boldsymbol{e}_{k}^{H} \boldsymbol{U}^{H} \boldsymbol{G}_{\mathrm{post}} \boldsymbol{\Pi} \boldsymbol{\boldsymbol { G } _ { \mathrm { pre } }} \boldsymbol{U} \boldsymbol{e}_{k}\right) .
\end{aligned}
$$

Based on (32) and (34), the SINR $\gamma_{k}$ can be expressed as

$$
\gamma_{k}=\frac{\left|E\left[\hat{d}_{k}\right]\right|^{2}}{\sigma_{k}^{2}}=\frac{\boldsymbol{e}_{k}^{H} \boldsymbol{U}^{H} \boldsymbol{G}_{\mathrm{post}} \boldsymbol{\Pi} \boldsymbol{G}_{\mathrm{pre}} \boldsymbol{U} \boldsymbol{e}_{k}}{1-\boldsymbol{e}_{k}^{H} \boldsymbol{U}^{H} \boldsymbol{G}_{\mathrm{post}} \boldsymbol{\Pi} \boldsymbol{G}_{\mathrm{pre}} \boldsymbol{U} \boldsymbol{e}_{k}} .
$$

Upon applying $\boldsymbol{G}_{\text {post }}=\left(\boldsymbol{G}_{\text {pre }}^{H} \boldsymbol{\Pi} \boldsymbol{G}_{\text {pre }}+\sigma_{n}^{2} \boldsymbol{I}_{K}\right)^{-1} \boldsymbol{G}_{\text {pre }}^{H}$ from (6) into the preceding equation, we obtain

$\gamma_{k}=\frac{\boldsymbol{e}_{k}^{H} \boldsymbol{U}^{H}\left(\boldsymbol{G}_{\mathrm{pre}}^{H} \boldsymbol{\Pi} \boldsymbol{G}_{\mathrm{pre}}+\sigma_{n}^{2} \boldsymbol{I}_{K}\right)^{-1} \boldsymbol{G}_{\mathrm{pre}}^{H} \boldsymbol{\Pi} \boldsymbol{G}_{\mathrm{pre}} \boldsymbol{U} \boldsymbol{e}_{k}}{1-\boldsymbol{e}_{k}^{H} \boldsymbol{U}^{H}\left(\boldsymbol{G}_{\mathrm{pre}}^{H} \boldsymbol{\Pi} \boldsymbol{G}_{\mathrm{pre}}+\sigma_{n}^{2} \boldsymbol{I}_{K}\right)^{-1} \boldsymbol{G}_{\mathrm{pre}}^{H} \boldsymbol{\Pi} \boldsymbol{G}_{\mathrm{pre}} \boldsymbol{U} \boldsymbol{e}_{k}}$.

After some further simplification with the aid of the matrix inverse lemma, it can be shown that $\gamma_{k}$ can be expressed as

$$
\gamma_{k}=\frac{1}{\sigma_{n}^{2} \boldsymbol{e}_{k}^{H} \boldsymbol{U}^{H}\left(\boldsymbol{G}_{\mathrm{pre}}^{H} \boldsymbol{\Pi} \boldsymbol{G}_{\mathrm{pre}}+\sigma_{n}^{2} \boldsymbol{I}_{K}\right)^{-1} \boldsymbol{U} \boldsymbol{e}_{k}}-1 .
$$

Finally, upon completing the multiplications of the matrices invoked, we can obtain (7).

\section{APPENDIX B}

\section{MAXIMIZATION OF THE MiNiMUM}

\section{SIGNAL-TO-INTERFERENCE-AND-NOISE-RATIO}

For simplicity of description, we rewrite (17) as

$$
\gamma_{i}=a_{i} x_{i}+b_{i}, \quad i=0,1, \ldots, D-1
$$

where, when comparing (38) with (17), we have

$$
a_{i}=\frac{\psi_{i}^{-2}}{K_{i} \sigma_{n}^{2}} \quad x_{i}=\bar{\Omega}_{i} \quad b_{i}=\frac{\psi_{i}^{-2}}{K_{i}} \sum_{j \in \mathcal{F}_{i}} \frac{1}{\Pi_{j}}-1 .
$$

Therefore, the optimization objective is to find the solutions to $x_{0}, x_{1}, \ldots, x_{D-1}$, which maximize the minimum SINR expressed as

$$
\gamma_{\min }=\min \left\{\gamma_{0}, \gamma_{1}, \ldots, \gamma_{D-1}\right\}
$$

when given $\sum_{i=0}^{D-1} x_{i} \leq K$.
To solve the foregoing optimization problem, we first prove the following observation.

Observation 1: The minimum SINR $\gamma_{\min }$ of (40) can be expressed as

$$
\gamma_{\min }=\lim _{p \rightarrow-\infty}\left(\sum_{i=0}^{D-1} \gamma_{i}^{p}\right)^{1 / p} .
$$

Proof: Let $q=-p$. Then, (41) can be written as

$$
\gamma_{\min }=\frac{1}{\lim _{q \rightarrow \infty}\left(\sum_{i=0}^{D-1}\left(1 / \gamma_{i}\right)^{q}\right)^{1 / q}}
$$

where the denominator at the right-hand side denotes the max norm (or $l_{\infty}$ norm) of $1 / \gamma_{0}, 1 / \gamma_{1}, \ldots, 1 / \gamma_{D-1}$, which satisfies 1he property [25]

$$
\begin{aligned}
\lim _{q \rightarrow \infty}\left(\sum_{i=0}^{D-1}\left(1 / \gamma_{i}\right)^{q}\right)^{1 / q} & =\max \left\{\frac{1}{\gamma_{0}}, \frac{1}{\gamma_{1}}, \ldots, \frac{1}{\gamma_{D-1}}\right\} \\
& =\frac{1}{\min \left\{\gamma_{0}, \gamma_{1}, \ldots, \gamma_{D-1}\right\}} .
\end{aligned}
$$

Therefore, we have (41).

Furthermore, as shown in (41), for a given value of $p<0$, the value of $\left(\sum_{i=0}^{D-1} \gamma_{i}^{p}\right)^{1 / p}$ monotonically increases with the value of $\sum_{i=0}^{D-1} \gamma_{i}^{p}$. Hence, instead of maximizing $\left(\sum_{i=0}^{D-1} \gamma_{i}^{p}\right)^{1 / p}$, the optimization can be achieved by first minimizing $\sum_{i=0}^{D-1} \gamma_{i}^{p}$ and then letting $p \rightarrow-\infty$. Consequently, upon taking into account the constraint of $\sum_{i=0}^{D-1} x_{i} \leq K$ and (38), the original optimization problem can be alternatively solved by first finding the minimum of the augmented cost function

$$
J=\sum_{i=0}^{D-1}\left(a_{i} x_{i}+b_{i}\right)^{p}-\lambda\left(\sum_{i=0}^{D-1} x_{i}-K\right) .
$$

Upon taking the gradient of $J$ with respect to $x_{i}$ and equating the result to zero, we can obtain

$$
x_{i}=\left(\frac{\lambda}{p}\right)^{\frac{1}{p-1}} a_{i}^{-\frac{p}{p-1}}-\frac{b_{i}}{a_{i}}, \quad i=0,1, \ldots, D-1 .
$$

Substituting $x_{i}$ for $i=0,1, \ldots, D-1$ to the constraint $\sum_{i=0}^{D-1} x_{i}=K$ gives

$$
\left(\frac{\lambda}{p}\right)^{\frac{1}{p-1}}=\frac{\sum_{i=0}^{D-1}\left(b_{i} / a_{i}\right)+K}{\sum_{i=0}^{D-1} a_{i}^{-\frac{p}{p-1}}} .
$$

Then, substituting (46) into (45), one can obtain

$$
x_{i}=\frac{a_{i}^{-\frac{p}{p-1}}}{\sum_{j=0}^{D-1} a_{j}^{-\frac{p}{p-1}}}\left(\sum_{j=0}^{D-1} \frac{b_{j}}{a_{j}}+K\right)-\frac{b_{i}}{a_{i}}
$$

Finally, the optimum solution to $x_{i}$ can be obtained by letting $p \rightarrow-\infty$, which yields

$$
x_{i}=\frac{a_{i}^{-1}}{\sum_{j=0}^{D-1} a_{j}^{-1}}\left(\sum_{j=0}^{D-1} \frac{b_{j}}{a_{j}}+K\right)-\frac{b_{i}}{a_{i}}
$$


Furthermore, when substituting $x_{i}$ of (48) into (38), we obtain the resultant SINR

$$
\gamma_{i}=\gamma_{c}=\frac{\sum_{j=0}^{D-1} b_{j} / a_{j}+K}{\sum_{j=0}^{D-1} a_{j}^{-1}}, \quad i=0,1, \ldots, D-1
$$

which shows that the optimization results in the same SINR for different groups, i.e., $\gamma_{0}=\gamma_{1}=\cdots=\gamma_{D-1}=\gamma_{c}$.

\section{REFERENCES}

[1] K. Fazel and S. Kaiser, Multi-Carrier Spread-Spectrum and Related Topics. Norwell, MA: Kluwer, 2002.

[2] L.-L. Yang, Multicarrier Communications. Chichester, U.K.: Wiley, 2009.

[3] T. M. Lok and T. F. Wong, "Transmitter and receiver optimization in multicarrier CDMA systems," IEEE Trans. Commun., vol. 48, no. 7, pp. 1197-1207, Jul. 2000.

[4] Z.-Q. Luo, T. N. Davidson, G. B. Giannakis, and K. M. Wong, "Transceiver optimization for block-based multiple access through ISI channels," IEEE Trans. Signal Process., vol. 52, no. 4, pp. 1037-1052, Apr. 2004

[5] P. Fu and K. Chen, "Rate, sub-carrier, and power allocations for multicarrier CDMA with LMMSE multiuser detection," IEEE Trans. Wireless Commun., vol. 6, no. 5, pp. 1574-1580, May 2007.

[6] J. Wang and L. B. Milstein, "Multicarrier CDMA overlay for ultrawideband communications," IEEE Trans. Commun., vol. 52, no. 10, pp. 1664-1669, Oct. 2004.

[7] K.-C. Chen and S.-T. Wu, "A programmable architecture for OFDMCDMA," IEEE Commun. Mag., vol. 37, no. 11, pp. 76-82, Nov. 1999.

[8] C. Chang and K. Chen, "Frequency-domain approach to multiuser detection in DS-CDMA communications," IEEE Commun. Lett., vol. 4, no. 11, pp. 331-333, Nov. 2000.

[9] Z. Wang and G. Giannakis, "Wireless multicarrier communications: Where Fourier meets Shannon," IEEE Signal Process. Mag., vol. 17, no. 3, pp. 29-48, May 2000.

[10] Y. Zhou, J. Wang, and M. Sawahashi, "Downlink transmission of broadband OFCDM Systems-Part I: Hybrid detection," IEEE Trans. Commun., vol. 53, no. 4, pp. 718-729, Apr. 2005.

[11] S. Z. X. Cai and G. Giannakis, "Group-orthogonal multicarrier CDMA," IEEE Trans. Commun., vol. 52, no. 1, pp. 90-99, Jan. 2004.

[12] T. Berger and D. Tufts, "Optimum pulse amplitude modulation-I: Transmitter-receiver design and bounds from information theory," IEEE Trans. Inf. Theory, vol. IT-13, no. 2, pp. 196-208, Apr. 1967.

[13] A. Scaglione, P. Stoica, S. Barbarossa, G. B. Giannakis, and H. Sampath, "Optimal designs for space-time linear precoders and decoders," IEEE Trans. Signal Process., vol. 50, no. 5, pp. 1051-1064, May 2002.

[14] D. P. Palomar, J. M. Cioffi, and M. A. Lagunas, "Joint Tx-Rx beamforming design for multicarrier MIMO channels: A unified framework for convex optimization," IEEE Trans. Signal Process., vol. 51, no. 9, pp. 2381-2401, Sep. 2003.

[15] D. Tse, Fundamentals of Wireless Communication. Cambridge, U.K.: Cambridge Univ. Press, 2005.

[16] D. Z. Filho, L. Féty, and M. Terré, "A hybrid single-carrier/multicarrier transmission scheme with power allocation," EURASIP J. WCNC, vol. 1, no. 1, pp. 1-11, 2008.

[17] T. Liu and C. Yang, "A symbol-level FDE and spread-spectrum mode design for multi-code multiple access systems," in Proc. IEEE ICC, May 19-23, 2008, pp. 4337-4341.

[18] G. Caire, G. Taricco, and E. Biglieri, "Optimum power control over fading channels," IEEE Trans. Inf. Theory, vol. 45, no. 5, pp. 1468-1489, Jul. 1999.

[19] I. Cosovic and S. Kaiser, "A unified analysis of diversity exploitation in multicarrier CDMA," IEEE Trans. Veh. Technol., vol. 56, no. 4, pp. 20512062, Jul. 2007.

[20] Q. Chen, E. S. Sousa, and S. Pasupathy, "Multicarrier CDMA with adaptive frequency hopping for mobile radio systems," IEEE J. Sel. Areas Commun., vol. 14, no. 9, pp. 1852-1858, Dec. 1996.

[21] I. E. Telatar, "Capacity of multiantenna Gaussian channels," Eur. Trans. Telecommun., vol. 10, no. 6, pp. 585-595, Nov./Dec. 1999.

[22] I. Gradshteyn and I. Ryzhik, Table of Integrals, Series, and Products. New York: Academic, 1980.
[23] A. Goldsmith, S. A. Jafar, N. Jindal, and S. Vishwanath, "Capacity limits of MIMO channels," IEEE J. Sel. Areas Commun., vol. 21, no. 5, pp. 684 702, Jun. 2003

[24] X. Zhang, D. P. Palomar, and B. Ottersten, "Statistically robust design of linear MIMO transceivers," IEEE Trans. Signal Process., vol. 56, no. 8 , pp. 3678-3689, Aug. 2008.

[25] Y.-P. Cheng, K.-Y. Zhang, and Z. Xu, Matrix Theory, 2nd ed. Xi'an, China: Northwesten Polytechnical Univ. Press, 2002.

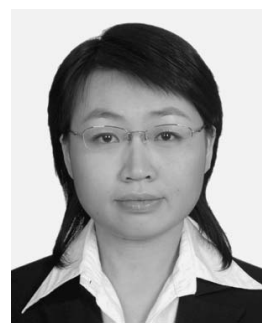

Tingting Liu (S'09) received the B.S. degree in 2004 from Beihang University, Beijing, China, where she is currently working toward the Ph.D. degree.

From December 2008 to January 2010, she was a Visiting Student with the School of Electronics and Computer Science, University of Southampton, Southampton, U.K. Her research interests are in the areas of wireless communications and signal processing, including multicarrier code division multiple access, multiple-input-multiple-output, and cooperative communications.

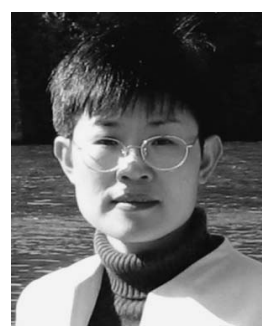

Chenyang Yang (SM'08) received the M.S.E. and $\mathrm{Ph} . \mathrm{D}$. degrees in electrical engineering from Beihang University (formerly the Beijing University of Aeronautics and Astronautics), Beijing, China, in 1989 and 1997, respectively.

She is currently a Full Professor with the School of Electronics and Information Engineering, Beihang University. She has published various papers and filed many patents in the fields of signal processing and wireless communications. Her recent research interests include signal processing in multiple-input-multiple-output, cooperative communication, cognitive radio, ultrawideband systems, and wireless sensor networks.

Dr. Yang is the Chair of the Beijing Chapter of the IEEE Communications Society. She has served as a Technical Program Committee Member for many IEEE conferences such as the International Conference on Communications and the IEEE Global Telecommunications Conference. She currently serves as an Associate Editor of the IEEE TRANSACTIONS ON WIRELESS COMMUNiCATIONS and an Associate Editor-in-Chief of the Chinese Journal of Communications and the Chinese Journal of Signal Processing. She was nominated as an Outstanding Young Professor of Beijing in 1995 and was supported by the First Teaching and Research Award Program for Outstanding Young Teachers of Higher Education Institutions by the Ministry of Education (P.R.C. “TRAPOYT”) during 1999-2004.

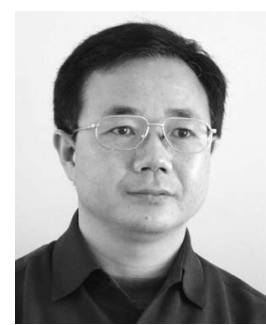

Lie-Liang Yang (M'98-SM'02) received the B.Eng. degree in communications engineering from Shanghai Tiedao University, Shanghai, China, in 1988 and the M.Eng. and Ph.D. degrees in communications and electronics from Northern (Beijing) Jiaotong University, Beijing, China, in 1991 and 1997, respectively.

From June 1997 to December 1997, he was a Visiting Scientist with the Institute of Radio Engineering and Electronics, Academy of Sciences of the Czech Republic, Prague, Czech Republic. Since December 1997, he has been with the University of Southampton, Southampton, U.K., where he is currently a Professor with the School of Electronics and Computer Science. He has published more than 240 research papers in journals and conference proceedings, authored/coauthored three books, and published several book chapters. Details about his publications can be found at http://wwwmobile.ecs.soton.ac.uk/lly/. His research has covered a wide range of topics in wireless communications, networking, and signal processing.

Dr. Yang is currently an Associate Editor of the IEEE TRANSACTIONS ON Vehicular Technology, the Journal of Communications and Networks, and the Security and Communication Networks journal. 